\title{
New developments in management of metastatic thyroid cancer
}

\author{
Jerome M. Hershman \\ Department of Medicine, David Geffen School of Medicine, University of California-Los Angeles, Los Angeles, CA 90073, USA.
}

Correspondence to: Prof. Jerome M. Hershman, Department of Medicine, David Geffen School of Medicine, University of California-Los Angeles, 11301 Wilshire Blvd, Los Angeles, CA 90073, USA. E-mail: jhershmn@g.ucla.edu

How to cite this article: Hershman JM. New developments in management of metastatic thyroid cancer. $J$ Cancer Metastasis Treat 2022;8:3. https://dx.doi.org/10.20517/2394-4722.2021.156

Received: 3 Aug 2021 Accepted: 9 Aug 2021 Published: 25 Jan 2022

Academic Editors: Lucio Miele, Alessandro Antonelli Copy Editor: Xi-Jun Chen Production Editor: Xi-Jun Chen

Thyroid cancer is increasing in prevalence ${ }^{[1]}$. Despite the use of ultrasonography for detection in an early phase, aggressive variants leading to metastatic disease continue to occur in perhaps $5 \%$ of patients ${ }^{[2]}$. This issue of the Journal of Cancer Metastasis and Treatment focuses on thyroid cancer. The ten timely review articles provide an excellent overview of selected aspects of metastatic thyroid cancer.

Davidson and Carr ${ }^{[3]}$ provide a thorough review of thyroid cancer metabolism and the principal pathways leading to thyroid cancer with superb illustrations of the various metabolic pathways. In addition, this article reviews the various pharmacologic inhibitors of the pathways, thus providing the reader with an efficient source for comprehending the extensive literature in this field.

Autophagy plays an important role in the homeostasis and survival of thyroid cells. Nagayama provides a very thorough review of the role of autophagy in thyroid cancer and defines the need for additional pharmacologic studies that target this pathway in thyroid cancer cells ${ }^{[4]}$.

Anaplastic thyroid cancer (ATC) is still a rapidly deadly tumor without any established therapy. Lee and Cheng ${ }^{[5]}$ present elegant studies of transcriptional pathways to explain transcriptional addiction that makes this tumor so difficult to treat effectively. They propose the use of small molecule inhibitors to target the transcriptional regulators in ATC. 
Quartuccio and Rubello ${ }^{[6]}$ performed a comprehensive database review and meta-analysis of ${ }^{18} \mathrm{~F}-\mathrm{FDG}$ PET/CT for detection of hematogenous spread of metastatic differentiated thyroid cancer and found a detection rate of $87 \%$ with specificity of $86 \%$. This confirms the utility of this technique in the follow-up of patients with metastatic DTC.

Corn et al. ${ }^{[7]}$ review the issue of finding uptake in the thyroid when ${ }^{18} \mathrm{~F}-\mathrm{FDG}$ PET/CT is performed for evaluation of other malignancies. They report incidental thyroid uptake on PET/CT at their institution in $3.4 \%$ of 4726 scans. Focal uptake was noted in $2.2 \%$, and diffuse uptake was diffuse in $1.2 \%$. They review the literature and provide insightful recommendations for the management of the incidentaloma.

Matrone et al. ${ }^{[8]}$ provide a thorough review of the scientific rationale and current therapy of metastatic medullary thyroid cancer.

Many patients with metastatic thyroid cancer are elderly and have cardiovascular disease that limits the choice of therapy for the metastases. Izkhakov et al. ${ }^{[9]}$ review the influence of cardiovascular disease in the overall prognosis among thyroid cancer survivors.

Damage to the recurrent laryngeal nerve is a major issue in the surgical treatment of thyroid cancer, especially in regard to second surgeries for removal of local metastases. Priya et al. ${ }^{[10]}$ provide a timely review of the methods and techniques of intraoperative recurrent laryngeal nerve monitoring for the preservation of the function of this key structure.

\section{DECLARATIONS}

\section{Authors' contributions}

The author contributed solely to the article.

\section{Availability of data and materials}

Not applicable.

\section{Financial support and sponsorship}

None.

\section{Conflicts of interest}

The author declared that there are no conflicts of interest.

\section{Ethical approval and consent to participate}

Not applicable.

\section{Consent for publication}

Not applicable.

\section{Copyright}

(c) The Author(s) 2022.

\section{REFERENCES}

1. Lim H, Devesa SS, Sosa JA, Check D, Kitahara CM. Trends in thyroid cancer incidence and mortality in the United States, $1974-2013$. JAMA 2017;317:1338-48. DOI PubMed PMC

2. Harari A, Singh RK. Increased rates of advanced thyroid cancer in California. J Surg Res 2016;201:244-52. DOI PubMed

3. Davidson CD, Carr FE. Review of pharmacological inhibition of thyroid cancer metabolism. J Cancer Metastasis Treat 2021;7:45. DOI 
4. Nagayama Y. Autophagy and thyroid cancer. J Cancer Metastasis Treat 2021;7:6. DOI

5. Lee WK, Cheng SY. Targeting transcriptional regulators for treatment of anaplastic thyroid cancer. $J$ Cancer Metastasis Treat 2021;7:27. DOI

6. Quartuccio N, Rubello D. Role of ${ }^{18}$ F-FDG PET/CT in detection of hematogenous metastases of advanced differentiated thyroid carcinoma: a systematic review and meta-analysis. J Cancer Metastasis Treat 2021;7:14. DOI

7. Corn S, Mitmaker E, Tabar R, Ciarallo A, How J. Incidental thyroid uptake on PET scanning: epidemiology, clinical significance, and management challenge. J Cancer Metastasis Treat 2021;7:41. DOI

8. Matrone A, Gambale C, Prete A, et al. Systemic treatment of advanced, metastatic, medullary thyroid carcinoma. $J$ Cancer Metastasis Treat 2021;7:23. DOI

9. Izkhakov E, Keinan-boker L, Stern N. Cardiovascular morbidity and mortality as a confounder of prognosis in thyroid cancer. $J$ Cancer Metastasis Treat 2021;7:24. DOI

10. Priya S, Garg S, Dandekar M. Intraoperative monitoring of the recurrent laryngeal nerve in surgeries for thyroid cancer: a review. $J$ Cancer Metastasis Treat 2021;7:70. DOI 